\title{
Event Tree as a Classification Model
}

\section{Jelena Djurovic, Danica Brzic, Tatjana Kaludjerovic Radoicic}

Faculty of Technology and Metallurgy, Belgrade University, Karnegijeva, Belgrade, Serbia

\section{Email address:}

jelenadj@tmf.bg.ac.rs (J. Djurovic), brzic@tmf.bg.ac.rs (D. Brzic), kaludjerovic@tmf.bg.ac.rs (T. K. Radoicic)

\section{To cite this article:}

Jelena Djurovic, Danica Brzic, Tatjana Kaludjerovic Radoicic. Event Tree as a Classification Model. Science Research.

Vol. 3, No. 3, 2015, pp. 53-57. doi: 10.11648/j.sr.20150303.12

\begin{abstract}
In this paper event tree model as qualitative support method was used. This is applied to fault tree analysis. Transfer and transformation facts and rules for problem solving were described. Method for knowledge extraction was presented. Mechanism of decision making and conclusions was implemented. Faults detection and classification were examined. That is significant for isolating all types of hazard and take appropriate steps to reduce and control these hazards.
\end{abstract}

Keywords: Fault Tree, Cause-Consequence, Classification, Rules Modeling, Acquisition

\section{Introduction}

If it goes back to the chief tasks of safety analysis methods can be placed in four groups, two relating to the identification of problems and two to their assessment. All formal methods can be considered as combinations of two methods, cause-consequence analysis, a combination of incident sequence analysis and fault tree analysis [1]-[5].

In the design of a new plant, they should come in at the detailed safety design phase, when most of the Piping and instrumentation diagram (PID) are ready [6]-[9]. These methods are also useful in the inspection of plants already built and on stream. The methods from this class incident sequence analysis and fault tree analysis examine links between faults, represent them graphically, and can assign probabilities to them. Incident sequence analysis starts with a single fault, and observes how it may develop. The working hypothesis is that every safety measure can succeed or fail with a certain probability.

The design and construction of safe plants calls for a highly structured and organized procedure clearly setting forth what has to be done by whom and in what way, and focusing on the creation and routing of documents. This is called a safety management system.

Knowledge bases can be understand as a special case decision support systems and the other hand knowledge bases present new stage in development evaluation step of information technologies. Knowledge base is the basic element of the expert system. Expression expert system is very often applied to program which use knowledge for behavior man-expert simulation, or whose function has some attributes man-expert behavior [1], [2]. It has power to learn from experience, general knowledge achievement, reconceptualization, analogy resonable, transfer knowledge from one domain to the other, flexibility and changeable approach for problem solution. Behind these, expert selecting alternative solutions, explaining its diagnosis as well as to learn from previously experiences adding knowledge base new elements which achieving during the problem solution [3],[4],[10].

Basic difference between expert systems and classical programs is that expert systems manipulate with knowledge and classical programs manipulate with data. Expert system has ability to solve complex problem which including uncertainty by information processing.

In this paper knowledge building for problem recognition and solving was developed.

\section{Knowledge Making Systems}

The two areas of model development and analysis are addressed through the discussion of generic simulation environment. The knowledge based simulation environment is an expression of some control law or cognitive theory. To the extent that the rule base is derived from set of assumptions about the environment and performance expectations, it is a belief system. However, in the existing form, the goals are not expressed and the underlying assumptions are not evident. Consequently, they are opaque to the analyst and cannot be directly applied to the learning process. When expressed in hierarchical form the relationship that exist between goals and subgoals provide a basis for relating overall goal based system 
performance to specific assumptions about the variability and contribution of the supporting subgoals. In this form, the belief system is a full expression of some control theory in that the system's relationship with the environment, as expressed in a set of feasible state conditions, can be related either in overall system performance measures to be relationships and the subgoals that support them.

In recent years, many applications of expert systems to simulation have evolved as a computer aided knowledge engineering tools. There exist considerable success in developing knowledge aided simulation systems. Intelligent simulation highlights to potential to meet with the demand. The technological advance in simulation has addressed the research interest of intelligent simulation [1]. The research and development in this disciplinary has continued for several years, and its effort has produced three types of intelligent simulation systems: single expert systems, coupling systems and integrated intelligent systems.

Single expert systems only process symbolic information, and provide assistance to system engineers in decision, making process for of- line simulation and modeling.

Coupling systems that couple numerical computation programs into expert system such that in can be used to solve engineering simulation problems.

Integrated intelligent systems are large intelligence integration environments, which can integrate different expert systems or numerical packages together to solve complex problems.

In the analysis and synthesis of engineering systems, simulation is a major technique. The traditional simulation techniques are algorithm based. They are often inflexible and provide limited means to the user. In fact, such techniques can not clearly simulate the dynamic behavior of the real processes. The segregation of the database, knowledge base and inference engine in the expert system allows us to organize the different models and domain expertise efficiently because each of these components can be designed and modified separately.

Presently expert systems are extensively developed in the research of intelligent simulation systems. Among the successful artificial intelligence applications, most of the expert systems are production systems. Production systems facilitate the representation of heuristic reasoning such that expert systems can be built incrementally as the knowledge of expertise increases. The expertise knowledge for the problem is described by a set of production rules. The typical production rule is described as IF (condition)....THEN (action). Inference engine in executor. It must determine which rules are relevant to a given knowledge base and select one of them to apply. This control strategy is called conflict resolution.

\section{Object Knowledge Acquisition}

Knowledge based system must represents information abstractly so that it can be stored and manipulated effectively. Although experts have difficulty formulating their knowledge explicitly as rules and other abstractions (Fig.1). They find it easy to demonstrate their expertise in specific performance situations. Schemes for learning abstract representations, or concepts, from examples to interact directly with systems to transfer their knowledge.

A functional approach to designing expert simulation systems was proposed many authors. They chose the differential games models is described using semantic networks. The model generation methodology is a blend of several problem solving paradigms, and the hierarchical dynamic goal system construction serve as the basis for model generation.

A functional approach to designing expert simulation systems was proposed many authors. They chose the differential games models is described using semantic networks. The model generation methodology is a blend of several problem solving paradigms, and the hierarchical dynamic goal system construction serve as the basis for model generation. Discrete event approach, based on the geometry of the games, can obtain the solution generally in much shorter time. Cooperation between systems is achieved through a goal hierarchy.

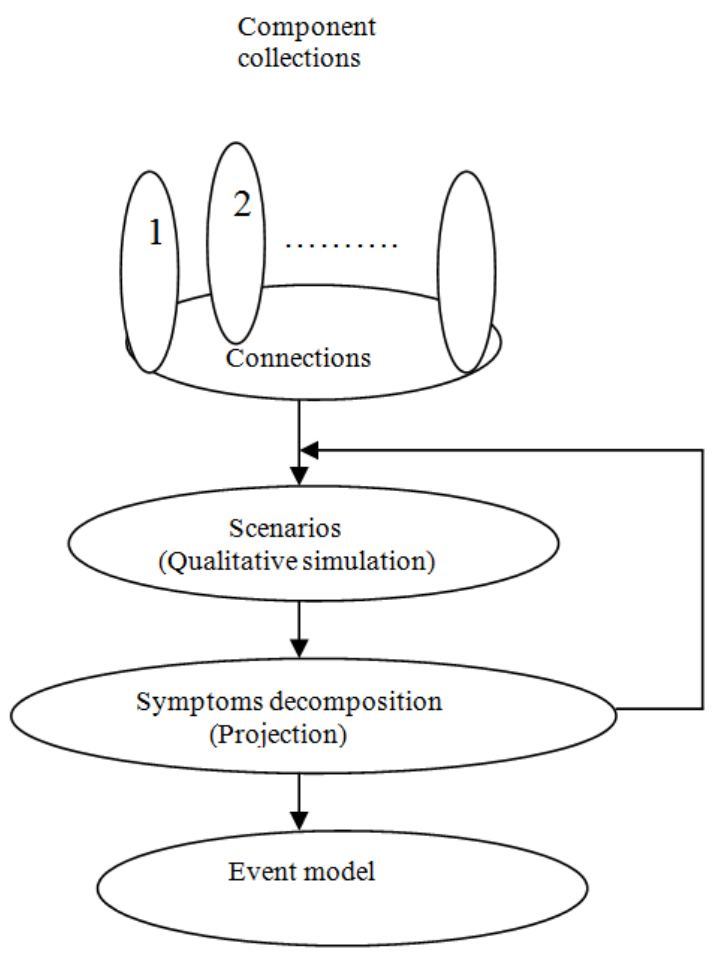

Fig. 1. Qualitative model.

Many expert systems have been introduced in such areas as medical diagnosis, chemical and biological reaction synthesis, pharmaceutical manufacturing, mineral and oil exploration, circuit analysis and equipment fault diagnosis.

These expert systems have emphasized the development of the knowledge acquisition process, the knowledge base, the inference procedure or control structure and maintaining the independence of each of 
these functions. Many days, computers have been widely used in simulation, but the use has been limited almost exclusively to purely algorithmic solutions. Many engineering problems are partial structured problems that deal with the non-numeric information and non-algorithmic procedure, and suitable for the application of artificial intelligence techniques. Expert systems provide programming methodology for solving non-structured problems which are difficult to be handled by purely algorithmic methods. The experience from building expert systems has shown that their power is most apparent when the considered problem is sufficiently complex.

In simulation, both qualitative and quantitative analysis are often applied together. Usually, qualitative decision efficiently made with symbolic and graphic information, and quantitative analysis is more conveniently performed by numerical information. Both methods often complement each other. Any numerical solution is only an approximation to the true solution, which is always represented analytically. Analytical solutions can only be obtained by symbolic processing.

\section{Qualitative State Processing}

The best way to solve complicated problem by expert systems is to distribute knowledge and to separate domain expertise. In such case, several expert systems may be used together. Each of them should be developed for solving a subdomain problem. Here, it is faced the problem of knowledge integration and management. Many expert systems can only be used alone for a particular purpose inflexibility. There are lack of coordination of symbolic reasoning and numeric computation, lack of integration of different expert system, lack of efficient management of intelligent systems and capability of dealing with conflict facts and events among the various tasks, being difficulty in modifying knowledge bases by end users other than the original developers.

Many integrated intelligent systems are a large knowledge environment, which consists of several symbolic reasoning systems and numerical computation packages. They are under the control of a supervising intelligent system, namely, meta-system. The meta system manages the selection, operation and communication of these programs.

The key issue to construct the integrated intelligent system is to organize a meta - system, which can thus be referred to as a control mechanism of meta level knowledge. Meta system has its data base, rule base and inference engine, but it decomposes its activities into the separated, strictly ordered, phases of information gathering and processing. The main functions of meta-systems are coordination all symbolic reasoning systems and numerical computation routine in an integrated intelligent systems, distribution knowledge into separate expert systems and numeric routines, acquiring new knowledge, finding a near optimal solution for the conflict solutions, and providing the possibility of parallel processing.

Most of the existing expert systems were developed for specific purposes. Usually, they were implemented with the symbolic language, and production rules were used to represent domain expertise. In light of application, such expert systems can only process symbolic information and make heuristic inference. Lack of numerical computation and uncoordinated single application make them very limited on the capability of solving the real engineering problems. Expert systems need data processing.

The coordination of symbolic reasoning and numerical computation is required heavily for simulation with expert systems. A few developers tried to develop expert systems with conventional languages. Other suggested to field expert systems in conventional languages, in order to achieve integration. Another disadvantage is that the procedural language environment cannot provide many good features that the symbolic language provides, such as easy debugging allowance for interruption by human experts.

Numerical languages often have a procedural flavor, in which the program control is command driven. They are very inefficient when dealing with processing strings. Symbolic languages are more declarative and data driven. However, it is very slow for symbolic languages to execute numeric computations. Complex problem can not be solved by purely symbolic or numerical techniques. Coupling of symbolic processing and numerical computing is desirable to use numeric and symbolic languages in different portion of software system. The coupled systems approach is often required when domain expertise is needed to provide the user suggestion or to direct the problem solving process. The most appealing approach is to achieve deep coupling of numerical and symbolic module representing the modules function, inputs, outputs, usage constraints. This allows the system to be applied to a wide range of problems, and makes it more robust.

\section{Process Fault Tree Building}

Plant builders and operators view plant safety in terms of the types of hazards arising from the process. This approach would have the advantage that the required safety could be achieved by the most advantageous technical resources in each instance. In addition to that, the plant orientated view would no longer require multiple specific regulations covering occupational safety and health, air and water pollution control, since one criterion for the plant would simultaneously take care of related problems in the other areas. Identify and correctly assess all types of hazard and take appropriate steps to reduce and control these hazards.

As a case study the fluid transport system shown in Fig.2 was used. The system for fluid transport consists of a tank, pump, valves and pipe.

The study of fault detection and diagnostics of the transport system is concerned with designing a system that can assist a human operator in detecting and diagnosing equipment faults in order to prevent accidents.

The system topology or component interconnections are defined by the process connections of the working process model (Fig.2). The level of aggregation is defined by the 
modular component interconnections which define propagation paths of attributes within the system. Initial research starting by phase of the development of a conceptual framework which facilitate the modular specification of models, and second phase the development of a logic framework which will permit object using attributes and simulation techniques to be linked into executable models.

In the plant is to be conceived for a process that has been safety optimized in this way, two analytical tasks, followed by the design tasks, and must still be performed. In carrying out these tasks, some of the steps may have to be done more than once, recursively, because any change in the system due to the new measures can introduce new danger sources. for this reason, and because the development of a process and the associated plant is done step by step, with concomitant advances in understanding a "holistic" procedure, segmented by time, technical specialty, and logical relationships, must be adopted in the development, design, construction, and operation of a chemical plant.

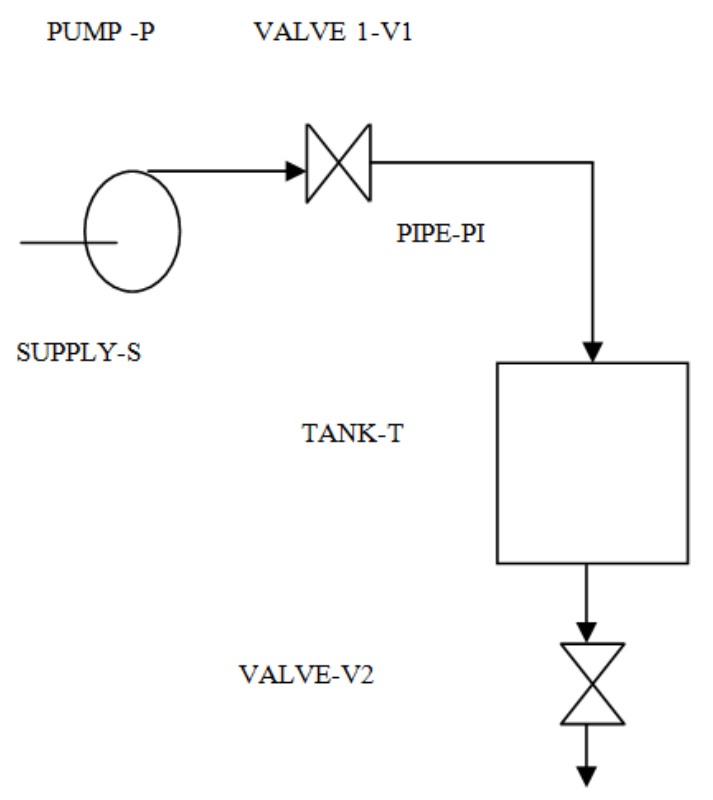

Fig. 2. Scheme of the transport fluid system.

The fault events of a system are in the first instance generally formulated in an IF-THEN form. This can be immediately reformulated using the operators AND, OR and NOT in Boolean form, if one can assume that the primary events have only two states existence and non-existence.

This system represent qualitative events model expressed by logic algebra, M, B, and L are independent logic variables representing the basic events malfunction, blockage and leakage, respectively.

Starting with the basic variables and their interrelations, the qualitative event model of the system can be formulated as shown in Fig.3.

To organize the logic of the rules, states variables must be defined within the system. Three types of state variables can be defined within a given component. The first are those variables whose values can be controlled by the system operator. These are controllable variables. The second set of state variables are those variables whose values are observable to the system operator. Last are those variables whose values are not immediately discernible by the system operator such as inner pressure, temperatures, and so on.

Scenarios are used to set initial states of the system state variables and attributes to predefined values prior to a model simulation run. This is necessary to evaluate "what if" scenarios concerning component malfunctions such as leakage due to worn or blockage due.

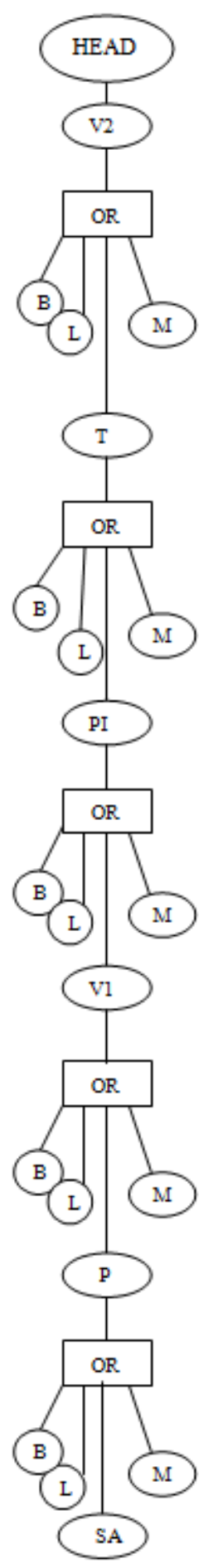

Fig. 3. The fluid transport pipe-line fault tree. 
A knowledge base building is consisting from the following steps: goals and subgoals definition, system identification, looking for identificators, state definition and rules statements, scenarios definition, for diagnostic purposed scenarios are evaluated by means of monitoring system symptom.

The symptom/scenario matrix displaying the final values of each symptoms which evolve from initial scenario states. Various rules can be applied to this system based on the transitivity relationships of the qualitative variables of the fluid flow. For instance, fluid pressure implies fluid supply and fluid flow implies fluid pressure. Thus, fluid flow implies fluid supply. But the same can not be said for fluid supply implying fluid flow. Each component provides attribution transformations depending upon its operating states.

Scenarios are used to set initial states of the system state variables and attributes to predefined values prior to a model simulation run. This is necessary to evaluate "what if" scenarios concerning component malfunctions such as leakage due to worn or blockage due.

The system can diagnose causes of faults associated with state variables pressure, flow rate and temperature, supply. The qualitative variables are described in three discrete values (low, medium, high). The following faults are considered blockage-B, leakage-L, malfunction or miss operation-M and supply absent-SA.

\section{Conclusion}

This paper considers diagnostic method and classification system building. The obtained results show power in problem knowledge acquisition as well as tree modeling, examining and reasoning.

For the fluid transport system a simple qualitative knowledge base was formed and logistic route within a decision tree was used.

The obtained results in this paper can be applied in the other domain.

\section{Acknowledgment}

The authors wish to express their gratitude to the Fund of Serbia for financial support.

\section{References}

[1] J.B.Savkovic--Stevanovic, Causes-effects functions, Comput. Ecol. Eng.,vol.8(2) 95-101, 2012, ISSN 1452-0729.

[2] J.B.Savković-Stevanović, Hazard recognition and quantifying, Comput. Ecol. Eng.,vol.9 (2) 63-71, 2013, ISSN 1452-0729.

[3] T.I.Oren and B.P.Zeiger AI in modelling and simulation direction to explore, Simulation vol., 56, 1988, pp.131-134.

[4] J. Savkovic-Stevanovic, Informatics, Faculty of Technology and Metallurgy Belgrade University, Belgrade, Serbia, 2007.

[5] F. S. Wong, W. Dong, M. Blanks, Couplig of symbolic and numerical computations on a microcomputer, Artificial Intelligence in Engineering, vol. 3, 1988,32-38.

[6] J.Savkovic-Stevanovic, A qualitative model for estimation for plant behavior, Comp. Chem. Eng., 18,1994,713-720.

[7] J.Savkovic-Stevanovic, Safety of the chemical processes, $\mathrm{p}$. 489-584, in the Book -Process plant operation, equipment, reliability and control,Eds.Michael Holloway, Chikezie Nwaoha,, and Oliver Onyewuenyi, John Wiley \&Sons,New York, April, ISBN 978-1-1180-2264-1,2012.

[8] J.Savkovic-Stevanovic et.al., Dictionary of Industrial Terms (Process Control, Reliability Eds., M. Holloway and C. Nwaoha, Scrivener Publishing LLC, Salem, New Jersey,U.S.A., 2013, ISBN 978-1-118-34457-6.

[9] J. Savkovic-Stevanovic., " Corrosion and Hazards “, p.517-659,of the Book: "Corrosion and Materials in Oil and Gas Industries, Editors, R. Javaherdashti, C.Nwaoha, H.Tan, CRC Press, NC, USA, april,2013.

[10] E.Turban, Decision support and expert systems, Management support systems, MPC, New York, 1990. 